\title{
Which Worldlines Represent Possible Particle Histories?
}

\author{
Samuel C. Fletcher \\ University of Minnesota, Twin Cities
}

\begin{abstract}
Based on three common interpretive commitments in general relativity, I raise a conceptual problem for the usual identification, in that theory, of timelike curves as those that represent the possible histories of (test) particles in spacetime. This problem affords at least three different solutions, depending on different representational and ontological assumptions one makes about the nature of (test) particles, fields, and their modal structure. While I advocate for a cautious pluralism regarding these options, I also suggest that re-interpreting (test) particles as field processes offers the most promising route for natural integration with the physics of material phenomena, including quantum theory.
\end{abstract}

\section{Events and Worldlines}

According to the general theory of relativity, the mathematical models of spacetime are, broadly construed, the Lorenzian manifolds $\left(M, g_{a b}\right) .{ }^{1}$ Each $M$ is a smooth, Hausdorff, connected, four-dimensional manifold, and each $g_{a b}$ is a smooth metric field of Lorentz signature $(1,3)$ on $M$. The points of $M$ are interpreted as events: structureless, fundamental happenings or potential happenings without temporal or spatial extension. The field $g_{a b}$ acts in many roles. In the present context, there are two most salient. First is its smooth partition of the elements $\xi^{a}$ of the tangent space at each point in $M$ into three classes: the timelike, null, and spacelike vectors (for which, respectively, $g_{a b} \xi^{a} \xi^{b}>0, g_{a b} \xi^{a} \xi^{b}=0$, and $\left.g_{a b} \xi^{a} \xi^{b}<0\right)$. Second is its assignment of a magnitude to each non-spacelike and spacelike $C^{1}$ curve $\gamma: I \rightarrow M$, with tangent vector $\xi^{a}$ and $I$ an open interval of $\mathbb{R}$, of the value $\int_{I}\left|g_{a b} \xi^{a} \xi^{b}\right|^{1 / 2} d s$. (Recall that a curve is (non-)spacelike when its tangent vector field is everywhere (non-)spacelike.) Typically, the piecewise smooth timelike curves are taken to be all and only the worldlines for possible (test) particles, representing their potential histories, and the (possibly broken) null geodesics (of the Levi-Civita connection compatible with $g_{a b}$ ) to be those of possible light rays. I shall provide some comments on each of these in turn.

\footnotetext{
${ }^{1}$ I have set aside the energy-momentum tensor $T_{a b}$; according to Einstein's field equation, it is determined according to $T_{a b}=\left(c^{4} / 8 \pi G\right)\left(R_{a b}-\frac{1}{2} R g_{a b}\right)$, where $c$ is the speed of light, $G$ is Newton's constant, and $R_{a b}$ and $R$ are, respectively, the Ricci and scalar curvatures associated with the Levi-Civita connection compatible with $g_{a b}$.
} 


\subsection{Events}

Events are the subject of interpretation not just in spacetime theories, but in metaphysics more generally. Philosophical accounts of events have taken that category as more expansive in content than is intended here, where "By an event we mean an idealized occurrence in the physical world having extension in neither space nor time" [20, p. 3]. Among traditional metaphysicians, by contrast events (potentially) encompass anything that happens, such as "Smiles, walks, dances, weddings, explosions, hiccups, hand-waves, arrivals and departures, births and deaths, thunder and lightning" [7]. By contrast with the structureless events represented by the points of a manifold, which have no proper parts, events in this broader sense include processes with positive duration that extend over space. That's not to say that there is consensus on what exactly delimits this content, or its metaphysical status in relation with spatiotemporal regions, properties, objects, processes, and facts - see Meyer $[33, \S 2.2]$ for a review of various positions - but rather that this content in any case outstrips those without temporal or spatial extension.

The concept of event as used in spacetime theories claims little with respect to most of these issues:

We use "event" as a neutral term here and intend no special significance. Some might prefer to speak, for example, of "equivalence classes of coincident point events" or "point event locations." [30, p. 119n1]

Traditional metaphysical accounts of events allows for distinct coincident (or overlapping) events. For example, one might describe the decay of a muon and the coincident emission of a muon neutrino as two separate but coincident events. By contrast, events for spacetime theories are associated with all mutually coincident events in this former sense.

We regard two events as being "the same" if they coincide, that is, if they "occur at the same place at the same time." That is to say, we are not concerned with how an event is marked - by firecracker or finger-snap-but only with the thing itself. [20, p. 4]

The decay of the muon and the emission of the muon neutrino would thus be "aspects" of the same event. (The quotations around "aspects" indicate that I do not intend to commit at this point to any metaphysical account of the relation between events and coincidence. I'll return to this issue in section 4.)

Conversely, traditional metaphysical accounts of events are less modally rich than those in spacetime theories. They assume that events, whatever they are, are existents: they exist as actual in some world or other. ${ }^{2}$ Importantly, and by contrast, an event within spacetime need not involve any material occurrences of particles at all, as long as it registers the possibility of an occurrence. On a first examination this may appear paradoxical: the merely possible occurrence that such an "empty" event registers is actual only in a different world. But

\footnotetext{
2 Forbes [17] is almost an exception: he takes the temporal order of a (non-relativistic) world to be modally constructed from actual events and their counterparts in "branching" counterpart worlds-ones who have initial segments of events that are perfect counterparts (in the sense of Lewisian counterpart theory [16, 29]). For Forbes [17], therefore, events are still fundamental world-bound existents, while times are modal constructions therefrom.
} 
how can something not actually existing be identical with or determine something actually existing? One resolution is to distinguish constitutive or identity relations from counterpart and representation relations. An event without material occurrences is not identical with one in another world therewith, but the two can be put in a correspondence via common representation by the mathematical model $\left(M, g_{a b}\right)$ (or by isomorphic such models) [15]. Many distinct general relativistic worlds can thus be represented by the same Lorentzian manifold $\left(M, g_{a b}\right)$. So to say that an event represented by a point in $M$ might, but might not, involve some material occurrence, is to affirm the equal adequacy of the mathematical model $\left(M, g_{a b}\right)$ to represent a world in which it does, and a world in which is does not.

Another resolution is to adopt a certain type of field ontology, which I discuss more in section 4. In any case, though, it seems that one cannot dispense with the modal features of these events in the context of general relativity. As Geroch [20, p. 4] reports, "Failure to do so in the present case would, as far as I can see, make further development of the theory virtually impossible."

\subsection{Worldlines}

The previous subsection focused on the interpretation of events in general relativity, revealing their modal character. Although events are represented by points of the manifold, I implicitly invoked the spacetime metric $g_{a b}$ when I maintained that these points can be understood as being without temporal or spatial extension. For it is the metric which endows certain collections of points a magnitude interpreted as physical extension in these senses.

This role for the metric, and the new modal considerations it raises in concert with those of events, is even more evident when one considers worldlines and what they represent. Following O'Neill [35, p. 11, ], let $\tilde{\gamma}: I \rightarrow M$, with $I$ an open interval of $\mathbb{R}$, be a smooth curve in a general relativistic spacetime $\left(M, g_{a b}\right)$.

Definition 1. A function $\bar{\gamma}:[a, b] \rightarrow M$, with $[a, b]$ a closed interval of $\mathbb{R}$, is called a curve segment when it has a smooth extension to a smooth curve, so that the tangent vector to $\bar{\gamma}$ can be uniquely defined at $\bar{\gamma}(a)$ and $\bar{\gamma}(b)$.

Definition 2. A function $\hat{\gamma}:[a, b] \rightarrow M$ is called a piecewise smooth curve segment when there is a finite ordered sequence $a=t_{0}<t_{1}<\ldots<t_{k+1}=b$ such that $\hat{\gamma}_{\mid\left[t_{i}, t_{i+1}\right]}$, called a piece of $\hat{\gamma}$, is a curve segment for each $i \in\{0,1, \ldots, k\}$.

(Note that any curve segment is a piecewise smooth curve segment.) The spacetime metric assigns magnitudes to piecewise smooth curve segments with tangent vector $\xi^{a}$ in the same way as to smooth curves, by integrating $\left|g_{a b} \xi^{a} \xi^{b}\right|^{1 / 2}$ over each segment and then summing the integrals together.

Definition 3. A function $\breve{\gamma}: I \rightarrow M$ is said to be a piecewise smooth curve when for any $a, b \in I$ such that $a<b, \breve{\gamma}_{\mid[a, b]}$ is a piecewise smooth curve segment.

(Note that any smooth curve is a piecewise smooth curve.) One says that $\breve{\gamma}$ contains the piecewise smooth curve segment $\breve{\gamma}_{\mid[a, b]}$.

Two further definitions are needed. 
Definition 4. A smooth curve or curve segment is said to be regular when its tangent vector never vanishes.

A piecewise smooth curve segment is regular just when its pieces are, and a piecewise smooth curve is regular just when every piecewise smooth curve segment it contains is regular.

Definition 5. A piecewise smooth curve segment $\hat{\gamma}$ is said to be oriented just in case whenever $\hat{\gamma}_{\left[t_{i}, t_{i+1}\right]}$ and $\hat{\gamma}_{\mid\left[t_{i+1}, t_{i+2}\right]}$ are two of its pieces, the right and left tangent vectors at $\hat{\gamma}\left(t_{i+1}\right)$ are co-oriented, i.e., their inner product via the metric $g_{a b}$ at this point is positive.

A piecewise smooth curve is oriented just when every piecewise smooth curve segment it contains is oriented.

Now let $\gamma$ be any oriented, regular, piecewise smooth curve or curve segment, and consider the interpretive principles for non-spacelike worldlines clearly stated by Malament [30, pp. 121-2]: ${ }^{3}$

(C1) $\gamma$ is timelike iff $\gamma[I]$ could be the worldline of a point particle with positive mass;

(C2) $\gamma$ can be reparameterized so as to be a null geodesic iff $\gamma[I]$ could be the trajectory of a light ray;

note in both cases the identification of a precise, mathematical class of objects - the images of certain curves - with physical states of affairs - worldlines and trajectories. Requiring that a curve be oriented, in the presence or regularity, ensures that the particle represented never spontaneously changes its local temporal orientation. ${ }^{4}$

To these interpretive principles Malament introduces several important qualifications and clarifications. "First, we are here working within the framework of relativity as traditionally understood and ignoring speculations about the possibility of particles that travel faster than light" [30, p. 121]. (I shall also ignore such speculations.) In addition, the point particles mentioned should be understood as test particles, those whose contributions to the energymomentum tensor $T_{a b}$ are neglected. This is, of course, an idealization, but an important one that lies at the heart of the interpretation of general relativity. The last clarification emphasizes the modality inherent in these interpretive principles and is worth quoting at length:

the modal character of the assertions (i.e., the reference to possibility) is essential. It is simply not true - take the case of $(\mathrm{C} 1)$ - that all images of smooth, timelike curves are, in fact, the worldlines of massive particles. The claim is that, at least so far as the laws of relativity theory are concerned, they could be. Of course,

\footnotetext{
${ }^{3}$ Malament assumes that $\gamma$ is just a regular smooth curve, but notes later that this might be relaxed for particles undergoing collisions, which is why I have introduced piecewise smooth curves and curve segments; this restriction indeed makes no substantive difference in understanding the following interpretive principles. I have also omitted Malament's footnotes and footnote symbols from the quotation to streamline the presentation.

${ }^{4}$ The role of regularity is to exclude such changes effected by buffering two non-co-oriented curve segments with a trivial (constant) curve, whose tangent vector vanishes. If one wanted to insist on the non-empty representational capacities of non-regular curves, then one could exchange regularity for a stronger and more complicated analog of orientation, but I shall not pursue such an approach here.
} 
judgments concerning what could be the case depend on what conditions are held fixed in the background. The claim that a particular curve image could be the worldline of a massive point particle must be understood to mean that it could so long as there are, for example, no barriers in the way. Similarly, in (C2) there is an implicit qualification. We are considering what trajectories are available to light rays when to intervening material media are present-i.e., when we are dealing with light rays in vacuo.

Though these ... concerns are important and raise interesting questions about the role of idealization and modality in the formulation of physical theory, they have little to do with relativity theory as such. Similar difficulties arise, for example, when one attempts to formulate corresponding principles within the framework of Newtonian gravitation theory. [30, pp. 121-2]

What's essential for the possible massive particles and light rays in question is that they can be supposed to interact in as specific a manner with other possible ambient matter as possible: their worldlines can be traced by the images of the appropriate curves without disturbing any other feature of the spacetime. In other words, they may be supposed to evade material interactions with other matter as dictated. This is another sense in which test bodies and test light rays are idealized.

Malament is right that idealization and modality plays important roles in physical theories generally, but I demur that there are no differences in general relativity compared with Newtonian gravitation. The aim of the following section is to show that in fact some novel issues $d o$ arise in relativity theory due to a phenomenon absent from standard Newtonian theory: closed timelike curves. This leads, in particular, to a re-evaluation of $(\mathrm{C} 1)$.

\section{Implications of Closed Timelike Curves}

\subsection{Branching Curves}

Definition 6. A piecewise smooth timelike curve or curve segment $\gamma: I \rightarrow M$ is said to contain a closed timelike curve (CTC) when there is a subset of $\gamma[I]$ homeomorphic to $S^{1}$; it is said to be a CTC when that subset is all of $\gamma[I] .^{5}$

Although many physicists - e.g., Hawking and Ellis [24, p. 189]-have expressed unease about spacetimes with CTCs on account of their leading to logical or causal paradoxes, I agree with the summary judgment of Earman [12, Ch. 6] that self-consistency of field solutions is already a feature of all models of general relativity, so the air of paradox has

\footnotetext{
5 There is some surprising variation in the definition of CTCs in the literature. O'Neill [35, p. 192] focuses on curve segments $\bar{\gamma}:[a, b] \rightarrow M$ that satisfy $\bar{\gamma}(a)=\bar{\gamma}(b)$ and are such that at these two points, their tangent vectors are non-zero and positively proportional. This rules out non-smooth CTCs. Earman [12, p. 165] assumes that spacetime is temporally orientable and takes a CTC to be a smooth, future-directed timelike curve (segment) from some $p \in M$ to itself. This rules out CTCs from existing in non-temporally orientable spacetimes. Perhaps there are sound arguments for either of these restrictions, but for present purposes they are not needed.
} 
been significantly overblown. ${ }^{6}$ Perhaps the way these "consistency conditions" manifest in particular models of the theory is of interest for further research, but I shall take them for granted in what follows because my discussion is not so dispositive towards any details about them.

Indeed, the implications to which I wish to draw attention arise not from CTCs per se, but from certain curves that contain CTCs but are not CTCs themselves. These curves involve a sort of branching that, given other common interpretative commitments of relativity theory, entails a kind of conceptual inconsistency. They fall into (at least) one of the following two classes.

Definition 7. A piecewise smooth timelike curve segment $\gamma:[a, b] \rightarrow M$ is right-branching when it contains smooth segments $\gamma_{1}:\left[a_{1}, b_{1}\right] \rightarrow M$ and $\gamma_{2}:\left[a_{2}, b_{2}\right] \rightarrow M$ and there is a number $s_{0} \in\left[0, \min \left\{b_{1}-a_{1}, b_{2}-a_{2}\right\}\right)$ such that $\gamma_{1}\left(a_{1}+s\right)=\gamma_{2}\left(a_{2}+s\right)$ and $\left(d \gamma_{1} / d s\right)\left(a_{1}+s\right)=$ $\left(d \gamma_{2} / d s\right)\left(a_{2}+s\right)$ for $s \in\left[0, s_{0}\right]$ but $\gamma_{1}\left(a_{1}+s\right) \neq \gamma_{2}\left(a_{2}+s\right)$ for $s \in\left(s_{0}, \min \left\{b_{1}-a_{1}, b_{2}-a_{2}\right\}\right]$. A piecewise smooth timelike curve is right-branching when it contains a right-branching piecewise smooth timelike curve segment. ${ }^{7}$

Definition 8. A piecewise smooth timelike curve segment $\gamma:[a, b] \rightarrow M$ is left-branching when $\gamma(-s)$ is right-branching. ${ }^{8}$ A piecewise smooth timelike curve is left-branching when it contains a left-branching piecewise smooth timelike curve segment.

Both classes consist of curves that contain a CTC with a point at which the image of the curve is not locally homeomorpic to a line. In particular, the right-branching curves contain smooth segments that have the same initial point and direction, but different final points, while the left-branching curves contain smooth segments that have the same final point and direction, but different initial points.

Definition 9. A curve or curve segment said to be branching (simpliciter) if it is either right- or left-branching.

Example 1. Consider Minkowski spacetime $\left(\mathbb{R}^{4}, \eta_{a b}\right)$ with a global Lorentz chart $(t, x, y, z)$, and consider the following coordinate expression of a worldline $\gamma: \mathbb{R} \rightarrow \mathbb{R}^{4}$ :

$$
\begin{aligned}
& t(\tau)= \begin{cases}\tau & \text { if } \tau \leq 0, \\
(c / A) \sinh (A \tau / c) & \text { if } \tau>0,\end{cases} \\
& x(\tau)= \begin{cases}1 / A & \text { if } \tau \leq 0, \\
\left(c^{2} / A\right) \cosh (A \tau / c) & \text { if } \tau>0,\end{cases} \\
& y(\tau)=0, \\
& z(\tau)=0 .
\end{aligned}
$$

\footnotetext{
${ }^{6}$ Indeed, CTCs appear in a wide variety of general relativistic models used to describe parts of our universe, such as the interior of Kerr black holes, and (more speculatively) possibly certain wormhole solutions [12, pp. 168-169].

${ }^{7}$ Cf. the definition of bifurcate curves of the first kind in Hajicek [23, p. 158].

8 I use the terms "right-" and "left-branching" rather than "future-" and "past-branching" because there is no need for the curve's tangent vectors to be co-oriented with the temporal orientation of the spacetime into which they map, or even for that spacetime to have a temporal orientation at all.
} 
At first the particle is unaccelerated, i.e., its worldline is geodesic until the event labeled $(0,1 / A, 0,0)$, after which it begins to accelerate at a constant rate $A$ in the $x$-direction. The differences in the parameterization $\tau$ at different events records the particle's elapsed proper time, and $c$ is the speed of light.

Considering the group $\mathbb{Z}$ which can act on the spacetime by temporal translation (i.e., along $t$ ), one can quotient by its group action to produce a Minkowski-like spacetime, "rolled up" along the $t$-direction, in which each $t$-slice has been identified with each $t+k$-slice, for every $k \in \mathbb{Z}$. Doing so, the resulting action of this quotient on $\gamma$ yields a right-branching smooth timelike curve, since, e.g., the curve segments $\gamma_{1}=\gamma_{\mid[-1,0]}$ and $\gamma_{2}=\gamma_{\mid[0,1]}$ satisfy $\gamma_{1}(-1)=\gamma_{2}(0)=(0,1 / A, 0,0)$ and $\left(d \gamma_{1} / d s\right)(-1)=\left(d \gamma_{2} / d s\right)(0)=(\partial / \partial t)^{a}$, while $\gamma_{1}(\tau-1)=$ $(0,1 / A, 0,0) \neq\left((c / A) \sinh (A \tau / c),\left(c^{2} / A\right) \cosh (A \tau / c), 0,0\right)=\gamma_{2}(\tau)$ for $\tau \in(0,1]$.

Letting $\beta: \mathbb{R} \rightarrow \mathbb{R}$ be defined as $\beta(\tau)=-\tau$ and using instead the curve $\gamma \circ \beta$ in Minkowski spacetime, the same construction procedure yields a left-branching smooth timelike curve.

This particular example is not locally homemorphic to the line at $(0,1 / A, 0,0)$ because it is "Y-shaped" there, although in general examples branching curves may also have points at which they are "X-shaped" or some other more general dendrite.

The sense in which branching curves entail a kind of conceptual inconsistency concerns the incompatibility of the following commitments:

1. The equation of motion for a massive test particle - Newton's second law-equates the net force $F^{a}$ it experiences at an event with the product of its mass $m$ and acceleration,

$$
F^{a}=m \xi^{b} \nabla_{b} \xi^{a}
$$

where $\xi^{a}$ is the tangent vector of its worldline at that event and $\nabla$ is the Levi-Civita derivative operator compatible with the spacetime metric. Thus on any spacetime region on which the force $F^{a}$ is defined (with appropriate smoothness), the initial value problem for a particle's worldline, both in the forward and backward directions, is well-posed; in particular, there is a unique maximally extended solution for each initial point and timelike tangent vector at that point [1].

2. Given a spacetime with a fixed collection of test particles worldlines within it, the force $F^{a}$ on a test particle at an event depends only on its intrinsic properties and its tangent vector at that event. In other words, there is a single net force that a particle with particular intrinsic properties and trajectory experiences at any event.

3. Timelike curves represent particle worldlines through their images. These divide up the events of spacetime into two classes: those at which the particle is present, and those at which it is not. That's to say that any two timelike curves with the same images have the same representational capacities [15] for massive particles: each of them represents equally well the possible worldline of a particle with particular instrinsic properties. This reflects in turn a commitment to the idea that the parameterization of a curve is just a convenient method of describing its image; particular useful parameterizations, such as those by arc length co-oriented with an ambient temporal orientation, should in fact be understood as induced on the curve image by the metric. 
The third commitment requires that, if a test particle is present at an event, then its only feature that can vary at that event is its trajectory (tangent vector). That means in particular that its intrinsic properties at that event are fixed. The second commitment then entails that because they are fixed, that particle experiences a determinate net force at that event which depends only on its trajectory there. It then follows from the first commitment that once the particle's trajectory is fixed at that event, so is its entire worldline. Yet, for branching curves, this is not so: a particle with the same intrinsic properties and initial or final trajectory at some event has at least two distinct worldline segments at which that event begins or ends, respectively. To be clear, there is no mathematical contradiction here: the incompatibility arises from the union of the mathematics of relativity theory with its interpretive principles, in particular those about the representational structure of timelike worldlines.

\subsection{Clarifications and Elaborations}

Before continuing in section 3 to outline three substantive resolutions to this inconsistency, each with its implications for the foundations of relativity theory, I would like to provide some elaborations on each of the three above commitments, from which together conceptual inconsistency arises, to forestall some possible initial objections. Indeed, each of the three requires some qualification, although in most cases relaxing these qualifications plausibly would not render the three commitments consistent.

For instance, in the first commitment, which involves the uniqueness of solutions to Newton's second law, I have set aside considerations involving test particles with instrinsic angular momentum, and how that may affect their equation of motion. Yet if those considerations were re-introduced, they would not mollify the conflict, for once the angular momentum of a particle is specified in addition to its trajectory at an event, similar uniqueness theorems for the initial value problem apply [31, 36, 10].

I have also assumed that any net force considered, and the spacetime metric itself, are sufficiently smooth to satisfy the preconditions of application of these uniqueness theorems. When those conditions are relaxed, it is possible even for there to be uncountably distinct geodesics passing through a point with a fixed timelike tangent vector there [14]. That said, it is not clear that relaxing these conditions would mollify the inconsistency of the three commitments. Even if a particle's worldline were not determined from its initial data at a point, the third commitment seems to demand that there must be a fact of the matter about what happens to the particle at that event: it follows one worldline through it or another, but whichever it does numbers exactly one. Otherwise the particle would be undergoing "fission" in the sense discussed in the philosophical literature on the identity of ordinary objects over time $[19, \S 4.7]$ : one particle evolves into two copies with the same intrinsic properties (or perhaps into one particle that is multilocated [21, §6], depending on one's metaphysics). But if a particle can undergo fission while its worldline contains a CTC, it is unclear why it couldn't when it doesn't. To model this requires abandoning the idea that woldlines are represented by the images of curves - maps from some real interval into the spacetime manifold - instead of the images of (say) an arbitrary dendrite. Finally, although we are considering test particles, countenancing their fission seems to violate the spirit of energy conservation.

As an aside: The considerations of the prior paragraph reveal why the conceptual incon- 
sistency arises for test particles and not light rays. When there is a unique solution to the initial value problem, the branching phenomenon for a worldline can only arise if the different branches experience different forces. In particular, if one is a geodesic, the other cannot be. ${ }^{9}$ While non-geodesic timelike curves can represent test particles undergoing forced motion, non-geodesic null curves cannot be interpreted as free light rays at all. ${ }^{10}$ Because there are no representational commitments with non-geodesic null curves, they cannot figure in an analogous argument about conceptual inconsistency.

Turning now to the second commitment, one sees that it amounts to the observation that forces on test particles are always single-valued. This is hardly controversial, and coheres with the prohibition on test particle fission adumbrated above. Perhaps two additional comments on it are in order. First, that the net force at an event is unique is entirely compatible with the nonlinear nature of the Einstein field equation. When a spacetime metric is given, supposing that certain timelike curves do or do not represent test particles does not affect this, since by definition these particles do not contribute to the energy-momentum tensor. Second, this force doesn't need to be a local function (although most considered in physics are), and may depend on the existence of other test particles, their properties, and the relations between them. But once these are fixed, the net force that a particle experiences should be uniquely specified.

The third commitment is perhaps the most subtle of the three. It is a commitment both about scientific representation, and the metaphysics of test particles. In the first place, it takes the image of a timelike curve to contain all the representational structure of that curve: two curves with the same images have the same representational capacities [15], and further information about those curves - their parameterizations - does not make them better representations of test particle, because there is no further structure thereby imputed that was not already with the image of the curve itself. In the second place, test particles maintain their intrinsic properties - mass, charge, etc.- for their whole worldlines. That the worldline of a particle contains a certain event means that the particle's properties at that event are given once and for all: there is no sense in which the event is doubly occupied by the particle with different properties, except perhaps when it has different trajectories through that event (although even that might be disputed), as would be modeled by a CTC with a kink. Put another way, a test particle can only interpenetrate itself-or, different temporal stages of a particle can only interpenentate - when distinct instantaneous velocities are involved.

When the particles's worldline extends in both directions of its parameterization beyond an event of interpenetration, forming an "X-shape," a question may arise regarding how from the image of the curve alone one can describe whether the particle passed through itself at this event, or whether it lead to a collision. However, unless the kinematics for the test particle - that is, a specification of disposition to rebound from particles of the same type - are given separately, this question is simply ill-posed under this third commitment. There is no fact of the matter whether there was a collision because such information is not represented in the image of the curve representing the worldline of the particle. One's mental

\footnotetext{
${ }^{9}$ In addition, given any coordinate system containing the branching, at least one cannot be described as an analytic function in those coordinates.

${ }^{10}$ I emphasize that these must be "free" light rays because of course the presence of refractive media can make a difference - cf. the long quotation by Malament in section 1.2.
} 
picture of collisions typically involves extended objects with different properties, while the allegedly possible collisions in the case at hand involve point particles with the same intrinsic properties - and not even different point particles, at that! Of course, if the allowance for interpenetration were curtailed to avoid these issues, it would not ameliorate the conceptual inconsistency; thus, it is not an essential aspect of the third commitment. Nevertheless, this commitment might be challenged on other grounds, which two of the resolutions described in the next section do.

\section{Three Resolutions}

In this section I consider three possible resolutions of the conceptual inconsistency revealed in the previous section. The first accepts the three commitments but restricts interpretive principle (C1) . The second modifies the third commitment to allow for test particles to have more structure than is represented by the image of a timelike worldline and the assignment of intrinsic properties thereto. The third rejects an aspect of the first commitment by dropping ontological commitment to test particles altogether in favor of fields. This allows, in principle, for the kind of field distribution that one might construe as "particle fission" under an idealized description. Each of these has implications for the foundations, interpretation, or ontology of relativity theory, the lattermost of which I discuss in the concluding section 4. Although I see the third approach as the most promising, I do not have conclusive arguments against the other two. So, instead of advocating for a single resolution above the others, I urge instead for further research.

\subsection{Acceptance}

The first resolution is simply to accept the three commitments leading to conceptual inconsistency, but deny that branching curves can represent the worldlines of possible massive test particles. This is, in a sense, the most ontologically conservative resolution, for the only modification that it makes to the interpretation of general relativity is a restriction of interpretative principle (C1). Namely, given an oriented, regular, piecewise smooth curve or curve segment $\gamma$,

$\left(\mathbf{C 1}^{*}\right) \gamma$ is timelike and non-branching iff $\gamma[I]$ could be the worldline of a point particle with positive mass.

Since the worldlines newly excluded by this restricted interpretive principle constitute only a strict subset of the curves containing CTCs, it will be attractive to those wary of CTCs in the first place. That said, the repercussions of this change throughout the theory need further exploration. Are there any theorems about models of general relativity, or particular constructions or calculations, that depend on the existence of branching timelike curves?

\subsection{Structured Test Particles}

The second resolution is to deny part of the third commitment leading to conceptual inconsistency, namely that the image of a timelike curve contains all the representational structure 
of that curve, and hence that massive text particles must maintain their intrinsic properties across their whole worldline. In particular, it affirms that the parameterization of a timelike curve represents more detail about a particle worldline than the image of the curve alone. It does not deny that identical images of timelike curves have the same representational capacities, but asserts rather that different timelike curves with the same image can in fact have inequivalent representational capacities. This is the sense in which this resolution adds additional structure to test particle worldlines.

In more detail, two parameterized timelike curves are considered representationally equivalent on this proposal just when they both have the same image, and can be reparameterized by arc length so that their domains are related by a constant shift. In more formal detail, $\gamma_{1}: I_{1} \rightarrow M$ and $\gamma_{2}: I_{2} \rightarrow M$ are representationally equivalent in the spacetime $\left(M, g_{a b}\right)$ if and only if $\gamma_{1}\left[I_{1}\right]=\gamma_{2}\left[I_{2}\right]$ and there exist diffeomorphisms $\alpha_{1}: I_{1}^{\prime} \rightarrow I_{1}$ and $\alpha_{2}: I_{2}^{\prime} \rightarrow I_{2}$ such that $\gamma_{1} \circ \alpha_{1}$ and $\gamma_{2} \circ \alpha_{2}$ are parameterized by their arc length according to $g_{a b}$, and there exists a constant $a \in \mathbb{R}$ such that $I_{1}^{\prime}+a=\left\{(b+a) \in \mathbb{R}: b \in I_{1}^{\prime}\right\}=I_{2}^{\prime}$. By contrast, according to the third commitment, only the first condition (of equality of images) is employed for representational equivalence.

For timelike curves not containing CTCs, the two characterizations of the representational capacities of timelike curves are equivalent. They only differ for curves containing CTCs. For instance, consider the smooth CTCs $\gamma_{1}:[0,1] \rightarrow M$ and $\gamma_{\infty}: \mathbb{R} \rightarrow M$ parameterized by arc length, whose shared image $\gamma_{1}[[0,1]]=\gamma_{\infty}[\mathbb{R}]$ is homeomorphic to $S^{1}$ and of magnitude 1 according to the spacetime metric. According to the third commitment, they are representationally equivalent, but not so according to the second resolution under discussion. According to this proposal, $\gamma_{1}$ represents a possible massive particle that "traverses" the smooth closed loop of its wordline once (before encountering its beginning and overlapping itself only at the single event $\gamma_{1}(0)=\gamma_{1}(1)$ ), while $\gamma_{\infty}$ "traverses" that loop countably infinitely many times, representing countably infinitely many interpenetrations with itself. Because now more of the structure of the curve's domain is representational, there are more possibilities for test particles. For example, the properties of a particle may change over distinct "traversals" of the same CTC. Because these properties may change, it is no longer the case that a particle with a given trajectory at an event must experience the same net force that an "earlier" or "later" state thereof does, despite their interpenetration at the same event. There is a tendency already to accept that for a CTC with a kink, the kink represents an event at which the particle interpenetrates itself with a different trajectory. The current proposal just extends the range of this acceptance to more properties and events.

Usually in a model of relativity theory, one understands all the worldly facts - facts about the spacetime and its represented "contents" - to be determined through the spacetime manifold and various fields thereon. The sense in which this is still true under the present proposal is a bit looser. Facts about, e.g., the time elapsed along a massive particle's wordline do not just depend on the events that worldline occupies, but how many times that event is occupied. However, the more times an event is occupied by a particle, the more strained does the test particle idealization seem. For curves such as $\gamma_{\infty}$ representing countably infinitely many massive text particles - or, perhaps more precisely, interpenetrations of temporal stages of the same immortal particle - at any point of its image, the idealization becomes essential to foreclose inconsistency. In particular, it is unclear what to make of such a situation if 
one takes test particles merely to be useful idealizations of fields. Taking seriously a field ontology indeed concerns the next resolution.

\subsection{Fields}

The third resolution is, in the first place, to deny part of the first commitment leading to conceptual inconsistency, namely the prohibition on particle fission. By itself, simply denying this prohibition will not provide resolution unless one also provides details about how doing so makes the usual uniqueness theorems for the equation of motion of a particle moot. What's added in this resolution is the replacement of a particle ontology with a field ontology, and the concomitant changes in the representational commitments that the formalism of relativity theory makes - in particular, the commitments made with curves. Because the second and third commitments make reference to particles, this resolution modifies them, too. If there are no particles, then the tangent vectors which enter into Newton's second law (equation 5) do not represent the trajectories of persisting particles, or really any persisting objects at all. Moreover, without particles one cannot attribute net forces to particles, either. Thus by denying that branching curves represent particles at all, the uniqueness claim for particle trajectories is unsupported by an existence claim. In a word, there is no contradiction in asserting that particles have unique solutions to their initial value problem, and that they can be represented by branching worldlines, if one denies that there are truly any particles at all. The seeming inconsistency, according to this resolution, arises solely from distortions introduced by idealizing certain field distributions as particles. Like with the first resolution, however, this requires some revision of interpretive principle (C1).

In more detail: According to a field ontology, matter in general and test particles in particular are represented by field distributions over spacetime points. Particles, as represented by the images of curves, are solely idealized objects, simplified surrogates for highly concentrated field distributions that, in local coordinate charts, can be approximated by timelike curve segments farther away from which the field is relatively vanishing. Thus, a branching worldline could well be a fine idealized representation of a field distribution whose concentration does indeed branch in spacetime (perhaps due to the interaction with and another field). This need not entail any incompatibility with energy-momentum conservation, as long as the equations of motion for the field (including perhaps its interactions with other fields) ensure its satisfaction.

With a field ontology, timelike curves can play at least two representational roles, the second involving more commitments than the first. First, they trace point-like processes within the field, the evolution of field values within the curve's image. The tangent vector of such a curve does not represent the four-velocity of the field or process in any significant sense; it rather just picks out how the events consituting the process described are related to one another in spacetime. That is, instead of being a dynamical property of a field, it is a part of the means of description for a process within the field. Analogously, one can describe a series of landmarks on a map by selecting one and a series of distance vectors that, when added sequentially to the initial landmark, pick out the rest of the series. The vectors are a means for describing the series of landmarks, but are not dynamical properties of some underlying material object to which one is committed.

A further representational role for curves in the context of a field ontology is for tracing 
not just any process within the field, but one around which the field is highly concentrated. (What technical conditions are needed for this I will leave to one side, since their details don't play a role in the argument here; see the discussion in Weatherall [46].) In this case, one may introduce test particles as a kind of emergent, idealized object in the theory. They are emergent in the sense that they are not found at all in a field ontology, and idealized in the sense that they can be described as limit objects of sequences of increasingly concentrated fields. But like with other idealized objects in physics - infinite planes, homogeneous fields, etc. - they do not abide by all the rules that non-idealized objects must [34]. That they do not raises no conceptual inconsistency for the rest of the theory.

For instance, it is sensible to describe the forces acting on a particle, even as an idealized object, when doing so is useful for prediction or explanation. In this sense commitment two can still hold for a field ontology, but only when the test particle idealization applies and only in this idealized sense. When it does not, the first commitment, Newton's second law (equation 5), is vacuous because tangent vectors are not defined for field distributions that cannot be idealized as curves. Most importantly, in the case of branching curves, no conceptual inconsistency arises because there is no sense in which the evolution of an extended field at an event depends on its changing momentum ("net force") there.

One advantage of this resolution is that it better coheres with our best theories of matter - field theories. Indeed, general relativity is hospitable for field-like matter and unfriendly to true particles. There is hardly any adequate way to de-idealize massive test particles into particles - that is, allow for them to contribute to the energy-momentum tensordue to the infinite concentrations of mass they suppose and the problems for dynamics this entails [46]. Rather, it seems better to consider them truly as field concentrations, as this resolution urges.

Yet that advantage also issues a challenge to the foundations of relativity theory. As was evident in interpretive principles (C1) and (C2), the interpretation of the mathematics of relativity theory is in terms of test particles. If one rejects test particles for fields, it would behoove one also to recast these interpretive principles in terms of fields. Fortunately, the two roles for test particles within a field ontology discussed above explain the delimited scope of efficacy of (C1) as an interpretive principle while also suggesting a modification. Namely, given an oriented, regular, piecewise smooth curve or curve segment $\gamma$,

$\left(\mathbf{C 1}^{* *}\right) \gamma$ is timelike iff $\gamma[I]$ could be a process of a matter field.

Which of those processes can be idealized as test particles will depends on one's precise criteria for approximation and localization. A process itself is a (piecewise) smoothly connected, locally linear causal sequence of events that register properties of the matter field - its field values.

Reinterpreting worldlines as field processes will require further reconceptualization of the foundations of relativity theory, but the suggestion is not unprecedented. For instance, Hawking and Ellis [24, p. 60] initially describe their interpretive postulate of "local causality" analogous to a combination of $(\mathrm{C} 1)$ and (C2) - in terms similar to those principles:

The equations governing matter fields must be such that if $\mathcal{U}$ is a convex normal neighborhood and $p$ and $q$ are points in $\mathcal{U}$ then a signal can be sent in $\mathcal{U}$ between $p$ and $q$ if and only if $p$ and $q$ can be joined by a $C^{1}$ curve lying entirely in $\mathcal{U}$, whose tangent vector is everywhere non-zero and is either timelike or null; 
the interpretive division of timelike and null curves arises from more direct consideration of relativistic kinematics and the optical limit of solutions to the Einstein-Maxwell equations, respectively. Instead of referring to test particles or light rays, however, they describe objects functionally and ambiguously in terms of "signals." They recognize and ameliorate the ambiguity immediately:

A more precise statement of this postulate can be given in terms of the Cauchy problem of the matter fields. Let $p \in \mathcal{U}$ be such that every non-spacelike curve through $p$ intersects the spacelike surface $x^{4}=0$ within $\mathcal{U}$. Let $\mathcal{F}$ be the set of points in the surface $x^{4}=0$ which can be reached by non-spacelike curves in $\mathcal{U}$ from $p$. Then we require that the values of the matter fields at $p$ must be uniquely determined by the values of the fields and their derivatives up to some finite order on $\mathcal{F}$, and that they are not unqiuely determined by the values on any proper subset of $\mathcal{F}$ to which it can be continuously retracted. [24, p. 60]

Signals or test particles have been replaced by fields, and the role of non-spacelike curves is to understand the causal connection between field values at an event and those at certain other collections of events - Cauchy surfaces, in particular. The sense in which the timelike and null directions are distinguished are not in terms of possible particle trajectories, but rather the directions of causal dependence in the sense given by the Cauchy problem. A process, represented by a timelike (or null) curve, is not an independently existing object in a spacetime but is rather a property or aspect of a matter field. This abides with similar suggestions concerning the application of the geodesic principle to true massive particles [46]. Exploring this idea further suggests natural corollaries for the ontology of spacetime events, which I explore in the next section.

\section{Implications for Ontology}

Among the three resolutions of the conceptual inconsistency presented in section 3, the first has no apparent ontological implications for relativity theory, but some representational implications: one doesn't change anything about test particles except for narrowing which curves can represent them. The second has minor ontological implications and moderate representational implications: particles have a greater range of properties than they could on the first resolution, and are represented by finer-grained equivalence classes of curves. The third resolution's implications are more substantial for ontology, but less for representation. All oriented, regular, piecewise smooth timelike curves and curve segments still play a representational role, but what they represent is different: processes of matter fields instead of histories of point test particles. These implications have synergistic correlates with the ontology of spacetime itself, which I had set aside in the introductory section. In this concluding section, I return to them before ending with how considerations from quantum theory impact the assessment of these resolutions.

\subsection{Spacetime}

If timelike curves are possible processes of matter fields, what is the relationship between these processes and events? More generally, what is the relationship between matter fields, 
processes, and events? Here I sketch two sorts of answers to these questions, answers which differ according to which sorts of entity they take as fundamental and which as derivative.

The first sort of answer makes processes and events more fundamental than matter fields. For instance, Whitehead $[49,50]$ and Russell $[42,43]$ take, in slightly different ways, matter to be participants in or properties of events. They do not however engage in detail with the question of how this ontological project interfaces with relativity theory. Mayr [32] and Romero [38, 37] do so more explicitly, the former taking processes as fundamental while the latter taking events instead. ${ }^{11}$ Of the two, only Romero [38] discusses substantively the relation between events, processes, and matter: objects are collections ("bundles") of events with particular shared properties. These events form the basic substance of the world [37].

This first sort of answer is essentially indifferent to whether one adopts a particle or field ontology for matter, since according to it, events maintain their modal properties of being possible sites of material coincidences, whatever form they may take. However, if one does adopt a field ontology a second answer becomes possible. According to this second answer, it is matter fields which are more fundamental than spacetime events, so that these events are, in a sense, properties of the fields. Many have made and rediscovered this suggestion $[45,2,3,40,41,9,47,48]$, with the events that all fields share sometimes called "pointcoincidences," often inspired by a famous quotation by Einstein [13, p. 155-6]: "Spacetime does not claim an existence of its own, but only as a structural quality of the field." Einstein's aspirations for a unified field theory distinguish his singular use of "field" and answers a potential objection, that the fact that all fields have the same event/coincidence properties goes unexplained. (If there is ultimately only one field, there is no coincidence to explain.) An additional advantage of this answer is that it involves a lower grade of modal commitment for events: no event is truly empty in a spacetime, merely a possible site for matter, but is instead always a property of some actual field (regardless of whether that field is explicitly modeled in the spacetime model). These advantages accrue whether one considers such an ontology as relational, which most making the suggestion have argued $[45,40,41,8,9,47,48]$, ultimately substantival, which other have objected [6], or not fitting neatly into either philosophical category $[2,3]$.

\subsection{Quantum Intimations}

Up until this point I have restricted attention to classical general relativity. There has been some conceptual advantage in doing so. In the first place, it is important to interpret our best theories with thoroughness unencumbered by the uncertain flux at the vanguard of research. Second, it evades the vexed question of a completely adequate theory of quantum gravity. But new theories also shine clearer interpretive light on hidden aspects of old theories, as general relativity has done with Newtonian gravitation [26], for example, or QED with classical electromagnetism [5]. So it is worth considering how the developments of quantum gravity and quantum theory more general bear on the three resolutions I have put forward.

For the most part, these developments favor the third resolution. Many of those advocating for a field ontology according to which spacetime is a property of the material field(s)

\footnotetext{
11 Whitehead, Russell, and Mayr are all concerned to construct simple events (i.e., those without parts) as, essentially, direct limits of series of nested processes. Their motivation comes, in part, from the empirical inaccessibility of such simple events. But for present purposes these differing motivations can be set aside.
} 
do so with arguments based in quantum mechanics [8], quantum field theory [2, 8, 9], or loop quantum gravity $[40,41]$. But one need not accept the second answer to the spacetime ontology question of the previous subsection to find support. Among those who take events to be ontologically more fundamental than matter also see fields, understood as properties of events, as more compatible with quantum mechanics [11], quantum field theory [22], and causal set theory $[38,37]$. Indeed, even those not principally concerned with spacetime ontology take our best theories of matter, quantum field theories, to provide for a field ontology, while any particle ontology (required by the first two resolutions) is highly problematic $[18,25]$. If our best interpretation of general relativity is to be compatible therewith, then the third resolution is thereby favored.

Not all agree however that our best physical theories favor fields over particles, whether for quantum field theory [4] or even classical field theory [28]. Those who do not have either of the first two resolutions at their disposal. So whatever ontological whispers one discerns and interprets from quantum theory, at least one of the three resolutions to the conceptual inconsistency in relativity theory remains viable. ${ }^{12}$

\section{References}

[1] Vladimir I Arnol'd. Ordinary Differential Equations. Springer, Berlin, 3rd edition, 1992. Trans. Roger Cooke.

[2] Sunny Y. Auyang. How Is Quantum Field Theory Possible? Oxford University Press, New York, 1995.

[3] Sunny Y. Auyang. Spacetime as a fundamental and inalienable structure of fields. Studies in History and Philosophy of Modern Physics, 32(2):205-215, 2001.

[4] David Baker. Against field interpretations of quantum field theory. British Journal for the Philosophy of Science, 60(3):585-609, 2009.

[5] Gordon Belot. Understanding electromagnetism. British Journal for the Philosophy of Science, 49(4):531-555, 1998.

[6] James Binkoski. Geometry, fields, and spacetime. British Journal for the Philosophy of Science, page axy002, forthcoming.

[7] Roberto Casati and Achille Varzi. Events. In Edward N. Zalta, editor, The Stanford Encyclopedia of Philosophy. Metaphysics Research Lab, Stanford University, winter 2015 edition, 2015.

[8] Dennis Dieks. Space and time in particle and field physics. Studies in History and Philosophy of Modern Physics, 32(2):217-241, 2001.

12 A further option suggested for quantum mechanics [44] and quantum field theory [27] is the tropebundle ontology - see Rossanese [39] for discussion. Because tropes are particularized properties, rather than instances of universals, they seem to fit roughly with those who take events to be more fundamental than matter, at least for the present purposes. 
[9] Dennis Dieks. Events and covariance in the interpretation of quantum field theory. In Meinard Kuhlmann, Holger Lyre, and Andrew Wayne, editors, Ontological Aspects of Quantum Field Theory, pages 215-234. World Scientific, Singapore, 2002.

[10] W. G. Dixon. Dynamics of extended bodies in general relativity. i. momentum and angular momentum. Proceedings of the Royal Society of London A, 314(1519):499-527, 1970.

[11] Mauro Dorato. Events and the ontology of quantum mechanics. Topoi, 34:369-378, 2015.

[12] John Earman. Bangs, Crunches, Whimpers, and Shrieks: Singularities and Acausalities in Relativistic Spacetimes. Oxford University Press, Oxford, 1995.

[13] Albert Einstein. Relativity: The Special and General Theory. Crown Publishers, New York, 1961.

[14] Samuel C Fletcher. Indeterminism, gravitation, and spacetime theory. In G. HoferSzabó and L. Wroński, editors, Making it formally Explicit: Probability, causality and indeterminism, pages 179-191. Springer International Publishing, Cham, 2017.

[15] Samuel C. Fletcher. On representational capacities, with an application to general relativity. Foundations of Physics, Aug 2018.

[16] Graeme Forbes. The Metaphysics of Modality. Oxford University Press, Oxford, 1985.

[17] Graeme Forbes. Time, events, and modality. In Robin Le Poidevin, editor, The Philosophy of Time, pages 80-95. Oxford University Press, Oxford, 1993.

[18] Doreen Fraser. The fate of 'particles' in quantum field theories with interactions. Studies in History and Philosophy of Modern Physics, 39(4):841-859, 2008.

[19] Andre Gallois. Identity over time. In Edward N. Zalta, editor, The Stanford Encyclopedia of Philosophy. Metaphysics Research Lab, Stanford University, winter 2016 edition, 2016.

[20] Robert Geroch. General Relativity from A to B. University of Chicago Press, Chicago, 1978.

[21] Cody Gilmore. Location and mereology. In Edward N. Zalta, editor, The Stanford Encyclopedia of Philosophy. Metaphysics Research Lab, Stanford University, fall 2018 edition, 2018.

[22] Rudolf Haag. On the sharpness of localization of individual events in space and time. Foundations of Physics, 43:1295-1313, 2013.

[23] P. Hajicek. Bifurcate space-times. Journal of Mathematical Physics, 12(1):157-160, 1971. 
[24] S W Hawking and G F R Ellis. The large scale structure of space-time. Cambridge University Press, Cambridge, 1973.

[25] Art Hobson. There are no particles, there are only fields. American Journal of Physics, 81(3):211-223, 2012.

[26] Eleanor Knox. Newtonian spacetime structure in light of the equivalence principle. British Journal for the Philosophy of Science, 65(4):863-880, 2014.

[27] Meinard Kuhlmann. The ultimate constituents of the material world. Ontos Verlag, Frankfurt, 2010.

[28] Dustin Lazarovici. Against fields. European Journal for Philosophy of Science, 8(2): $145-170,2017$.

[29] David Lewis. On the Plurality of Worlds. Basil Blackwell, Oxford, 1986.

[30] David B Malament. Topics in the Foundations of General Relativity and Newtonian Gravitation Theory. University of Chicago Press, Chicago, 2012.

[31] M. Mathisson. Neue mechanik materieller systeme. Acta Physica Polonica, 6:163-209, 1937.

[32] Dieter Mayr. A constructive-axiomatic approach to the Lie structure in general spacetime by the principle of approximative reproducibility. Foundations of Physics, 13(7): 731-743, 1983.

[33] Ulrich Meyer. The Nature of Time. Clarendon Press, Oxford, 2013.

[34] John D. Norton. Approximation and idealization: Why the difference matters. Philosophy of Science, 79(2):207-232, 2012.

[35] Barrett O'Neill. Semi-Riemannian Geometry, with Applications to Relativity. Academic Press, San Diego, 1983.

[36] A. Papapetrou. Spinning test-particles in general relativity. I. Proceedings of the Royal Society of London A, 209(1097):248-258, 1951.

[37] Gustavo Romero. On the ontology of spacetime: Substantivalism, relationism, eternalism, and emergence. Foundations of Science, 22(1):141-159, 2017.

[38] Gustavo E. Romero. A formal ontological theory based on timeless events. Philosophia, 44(2):607-622, Jun 2016.

[39] Emanuele Rossanese. Trope ontology and algebraic quantum field theory: An evaluation of Kuhlmann's proposal. Studies in History and Philosophy of Modern Physics, 44(4): 417-423, 2013.

[40] Carlo Rovelli. Halfway through the woods: Contemporary research on space and time. In John Earman and John D Norton, editors, The Cosmos of Science: Essays of Exploration, pages 180-223. University of Pittsburgh Press, Pittsburgh, 1997. 
[41] Carlo Rovelli. 'Localization' in quantum field theory: How much of QFT is compatible with what we know about space-time? In Tian Yu Cao, editor, Conceptual Foundations of Quantum Field Theory, pages 207-232. Cambridge University Press, Cambridge, 1999.

[42] Bertrand Russell. The Analysis of Matter. Kegan Paul, Trench, Trubner, London, 1927.

[43] Bertrand Russell. Our Knowledge of the External World. W. W. Norton, New York, 2nd edition, 1929/1914.

[44] Peter Simons. Particulars in particular clothing: Three trope theories of substance. Philosophy and Phenomenological Research, 54(3):553-575, 1994.

[45] Paul Teller. Space-time as a physical quantity. In Robert Kargon and Peter Achinstein, editors, Kelvin's Baltimore Lectures and Modern Theoretical Physics, pages 425-448. MIT Press, Cambridge, MA, 1987.

[46] James Owen Weatherall. Geometry and motion in general relativity. arXiv preprint arXiv:1810.09046, 2018.

[47] Hans Westman and Sebastiano Sonego. Events and observables in generally invariant spacetime theories. Foundations of Physics, 38(10):908-915, Oct 2008.

[48] Hans Westman and Sebastiano Sonego. Coordinates, observables and symmetry in relativity. Annals of Physics, 324(8):1585 - 1611, 2009.

[49] Alfred North Whitehead. The Concept of Nature. Cambridge University Press, Cambridge, 1920 .

[50] Alfred North Whitehead. Process and Reality. Free Press, New York, corrected edition, 1978/1929. Eds. David Ray Griffin and Donald W. Sherburne. 